https://doi.org/10.15407/ujpe65.2.120

F.M.A. AL-JOMAILY, M.I. KHALIL

1,2 Mosul University, College of Science

(Mosul, Iraq; e-mail: dr.firas@uomosul.edu.iq)

\title{
REVISITING TO THE GEIGER-NUTTAL RELATION TO BE EMPLOYED IN THE ESTIMATION OF THE HALF-LIVES OF SUPERHEAVY NUCLEI
}

\begin{abstract}
The half-lives for the even-even ( $e-e)$, even-odd ( $(e-o)$, odd-even (o-e) and odd-odd (o-o) nuclei in the range $100 \leqslant Z \leqslant 120$ have been tested within the Viola-Seaborg formula (VSF) and within the analytical formula of Royer (RF). We proposed another formula (Present Work Formula or PWF) with regard for the effect of angular momentum of the alpha decay particle and with the use of the relative neutron excess $\left(\frac{N-Z}{A}\right)$. Our formula includes a new set of parameters found by the least square fitting method of alpha decays of 128 nuclei. We obtained the standard deviations for each of the formulas for comparison. The results show an acceptable agreement with available data. The values of the suggested theoretical coefficient $(K)$ for the $P W F$ show a similar behavior of half-lives with $\alpha$-decay, which can be used to predict the new superheavy nuclei.

Keywords: Geiger-Nuttal, superheavy nuclei, alpha decay, half-lives, neutron excess ratio.
\end{abstract}

\section{Introduction}

One of the landmarks in modern physics, shaping the development leading to quantum mechanics, was the formulation of the empirical Geiger-Nuttal (GN) law in 1911 [1] concerning the partial half-life $\left(T_{1 / 2}\right)$ with alpha decay.

Recently, the amount of $\alpha$-decay data for heavy and superheavy nuclei has greatly increased $[2,3$, 4]. The universal formula for the alpha and cluster processes reproduces well the practical values for the half-lives for even and odd nuclei with $84 \leqslant Z \leqslant 100$ $[5,6]$. Within this procedure, the tunneling probability through the potential barrier was determined, by using the Wentzel-Kramers-Brillouin approximation. An acceptable accuracy was found in this field in comparison with other formulas [7, 8]. This idea was adopted by some authors to investigate the halflives of superheavy elements with alpha decay within the range of $100 \leqslant Z \leqslant 122$ [5]. Firas and Mayan [9] derived a semiempirical formula based on the GeigerNuttal rule and introduced some parameter for a single-body model with suitable constants obtained through the trial and error method. Their model involved the relative neutron excess $\left(\frac{N-Z}{A}\right)$ which is extremely important in calculations of $Q_{\alpha}$ and the half-

(C) F.M.A. AL-JOMAILY, M.I. KHALIL, 2020 live logarithm for even-even heavy nuclei. It is worth to note that the alpha decay model is usually used for the guessing of heavy and superheavy elements (SHE) $[10,11,12]$. Viola and Seaborg suggested an analytical relation for the guessing of half-lives, which is based on a Gamow-type formula [13]. A semiempirical relation was suggested by Poenaru and Ivascu [14] for the alpha decay fission theory for all groups of nuclei.

Moreover, the half-lives for e-e, e- $\mathrm{O}, \mathrm{O}^{-} \mathrm{e}$, and $\mathrm{o}^{-} \mathrm{O}$ nuclei with alpha decay in the range $52 \leqslant Z \leqslant 118$ have been tested within the Royer modified formula including new Royer coefficients obtained by the fitting of 356 isotopes [15]. Superheavy isotopes probe the extremes of the structure of nuclei with respect to the mass number of nuclei for a bound system. Their existence and the properties of the decay are one of the most essential challenges in nuclear physics $[16,17]$. Sayed \& ALmadar have estimated the alphadecay half-lives for all types of nuclei in the interval $Z=104-118$ according to the quantum mechanics theory (tunnel effect). Before the emission, the alpha particle moves inside the mother nucleus supposedly in a spherical field determined by the daughter nucleus. The lifetime of a nucleus with alpha-decay may give a rough measure of the extent to which the nuclear structure is capable of guessing the amount of

ISSN 2071-0194. Ukr. J. Phys. 2020. Vol. 65, No. 2 
nuclear density [18]. A very important results were achieved by Wang et al. [19], who studied many types of formulae in the field of superheavy elements. They found that the semi FFS2 formula is the best one for the prediction of the alpha-decay half-lives. In addition, the formulas UNIV2, VSS, and NRDX with their fewer coefficients have a well-done guessing of SHEs with alpha decay $[7,13,20,21,22,23]$. There is a research which explains that different technical coefficients do not alter significantly the transfer structure of fractional yields of medium heavy isotopes with regard for cluster half-lives [29]. A description of even-even $\mathrm{Pd}$ isotopes from $A=102$ to 106 in the framework of the interacting boson model was carried out in [30].

In this work, we intend to describe the $\alpha$-decay halflives with different proposed formulas. First, we apply the Viola-Seaborg-Sobiczewski approach which reveals the relationship between the alpha-decay $Q$ value and $T_{\alpha}(1 / 2)$ [13]. Second, we will use the analytical formula for the $\alpha$-decay half-lives constructed in [24]. We used the formula by Firas and Mayan [9] in a modified form to calculate the alpha-decay halflives with regard for the relation [9] that can be valid to all types of nuclei $\left(\mathrm{e}^{-} \mathrm{e}, \mathrm{e}^{-} \mathrm{O}, \mathrm{o}^{-} \mathrm{e}\right.$, and $\left.\mathrm{o}^{-} \mathrm{O}\right)$ in the interval $100 \leqslant Z \leqslant 120$. Eventually, the theoretical coefficient has been proposed for the prediction of new elements. This is done by solving the partial differential equation (8).

\section{VSF Tests for the Nuclei under Study}

Geiger and Nuttal proposed the following relation between the alpha-particle decay energy $(Q)$ and the alpha-decay half-lives $\left(T_{\alpha}\right)$ :

$\log _{10} T_{\alpha}=a+b Q_{\alpha}^{-1 / 2}$,

where the parameters $a$ and $b$ depend on the atomic number of a parent nucleus. In 1966, Viola and Seaborg utilized the Geiger-Nuttal formula and proposed the well-known Viola-Seaborg relation [13]:

$\log _{10} T_{\alpha}=a+b Q_{\alpha}^{-1 / 2}+\left(c Z+d+h_{\log }\right)$,

where $Z$ is the atomic number of the parent nucleus. $a, b, c, d$ are the coefficients that can be achieved by fitting the data given in [13]:

$a=1.66175, \quad b=-8.5166, \quad c=-0.20228$,

ISSN 2071-0194. Ukr. J. Phys. 2020. Vol. 65, No. 2 $d=-33.9069$

The quantity $h_{\log }$ is the hindrance factor for odd $-\mathrm{A}$ or odd-odd nuclei calculated by VSF:

$h_{\log }=0 \quad$ even-even nuclei,

$h_{\log }=0.772$ for odd-even nuclei,

$h_{\log }=1.066$ for even-odd nuclei,

$h_{\log }=1.144 \quad$ for odd-odd nuclei.

Equation (2) was applied to all nuclei in the range $100 \leqslant Z \leqslant 120$.

\section{RF Tests for the Nuclei Under Study}

The alpha-decay half-life can be evaluated suggesting that the incoming point is the contact point, and the outgoing point fits the value of the Coulomb energy with the practical $Q_{\alpha}$. The inertia coefficient is a miniature mass. Through this model of unified fission, the decay constant is simply the product of the number of collisions and the potential of penetration. There is no pre-modulation parameter [25, 26]. The relation between the $Q$ value of the alpha decay and half-lives suggested by G. Royer [24], by analyzing the process of alpha emission by a nucleus. This relation was applied to all nuclei $(\mathrm{e}-\mathrm{e}, \mathrm{e}-\mathrm{O}$, $\mathrm{o}^{-} \mathrm{e}$, and $\left.\mathrm{o}^{-} \mathrm{o}\right)$ in the interval $100 \leqslant Z \leqslant 120$.

For even-even nuclei

$\log _{10}\left[T_{1 / 2}(S)\right]=-25.31-1.1629 A^{1 / 6} \sqrt{Z}+\frac{1.5864 Z}{\sqrt{Q_{\alpha}}}$

For even-odd nuclei

$\log _{10}\left[T_{1 / 2}(S)\right]=-26.6-1.0859 A^{1 / 6} \sqrt{Z}+\frac{1.592 Z}{\sqrt{Q_{\alpha}}}$.

For odd-even nuclei

$\log _{10}\left[T_{1 / 2}(S)\right]=-25.68-1.1423 A^{1 / 6} \sqrt{Z}+\frac{1.592 Z}{\sqrt{Q_{\alpha}}}$.

For odd-odd nuclei

$\log _{10}\left[T_{1 / 2}(S)\right]=-29.48-1.113 A^{1 / 6} \sqrt{Z}+\frac{1.6971 Z}{\sqrt{Q_{\alpha}}}$.

Here, $Q_{\alpha}$ is the experimental value. 


\section{Present Work Formula (A New Approach)}

In our previous work [9], we proposed a semiempirical formula for even-even nuclei in the interval of $82 \leqslant$ $Z \leqslant 102$, in the following form:

$\log T=\frac{1.65\left(Z_{p}-2\right)}{\sqrt{Q_{\alpha}}}-26.6-$

$-\sqrt{\left[(1.08)(A-4)^{1 / 3}+2\right]\left(Z_{p}-2\right)}+\left(\frac{N-Z_{p}}{A}\right)$.

This formula (semiempirical relation) is based on the Geiger-Nuttal rule and involves some parameters of the single-particle model such as the radius of nucleus represented by $\left(1.08 A_{d}^{1 / 3}+2\right)$ and the atomic number of the daughter nucleus with their suitable constants that were obtained by the trial and error method. Moreover, the model contains the term representing the relative neutron excess $\left(\frac{N-Z}{A}\right)$, which is extremely important for the suitability of calculations of the half-life logarithm and its matching the experimental value. In this work, relation (7) becomes no longer valid to all types of nuclei under study. So, we introduce a more accurate general formula. This is done by adding two additional terms to the $l$-dependent formula in order to determine the alpha-decay half-lives of the even-even, even-odd, odd-even, and odd-odd nuclei. The formula involves also $A, Z, N$ of the mother nucleus, experimental decay energy $Q_{\alpha}$, and angular momentum $l$. The alphaparticle carries the angular momentum $l \neq 0$ for oddodd and odd-A nuclei in the ground-state transition which depends on the spin and parity of the parent and daughter nuclei. The minimum angular momentum mainly carried by the alpha particle with regard for the selection rules is zero $(l=0)$ for the ground state of even-even nuclei in view of their spin and parity [26]. As a result, the modified formula reads

$$
\begin{aligned}
& \log T=a \frac{\left(Z_{p}-2\right)}{\sqrt{Q_{\alpha}}}-b- \\
& -\sqrt{\left[(1.08)(A-4)^{1 / 3}+2\right]\left(Z_{p}-2\right)}+\left(\frac{N-Z_{p}}{A}\right)+ \\
& +c \frac{A N Z(l(l+1))^{1 / 4}}{Q}+d A\left[1-(-1)^{l}\right]
\end{aligned}
$$

where $Q$ is the alpha-decay energy given in $\mathrm{MeV}$ units, and $A, Z$, and $N$ are the mass, charge and the number of neutrons of the mother nucleus, respectively. The parameters $a, b, c$, and $d$ are obtained using the least square fitting of $\alpha$-decay data of the studied nuclei. Taking the last two terms from [27], we get the following.

For even-even nuclei

$a=1.65, \quad b=-27.8, \quad l=0$,

$c=1.4948 \times 10^{-6}, \quad d=10 \times 10^{-4}$.

For even-oddb nuclei

$a=1.662, \quad b=-28.8, \quad l=3$,

$c=8.3678 \times 10^{-4}, \quad d=2.343 \times 10^{-6}$.

For odd-even nuclei

$a=1.64, \quad b=-27.4, \quad c=1.9003 \times 10^{-6}$,

$d=12 \times 10^{-6}, \quad l=3$.

For odd-odd nuclei

$a=1.66, \quad b=-26.6, \quad c=$ zero,$\quad d=$ zero,$\quad l=3$.

Equation (8) was applied to all nuclei in the interval $100 \leqslant Z \leqslant 120$.

\section{Proposing a Theoretical Coefficient for the Prediction of New Elements}

It is known that $Q_{\alpha}$ is of importance for calculating $T_{1 / 2}$ of the alpha decay. Up to now, there was no theoretical formula that could describe accurately the alpha-decay energy with a deviation less than $0.5 \mathrm{MeV}$ and reach the guessing of half-lives with an acceptable accuracy. To avoid this difficulty, we introduce the quantity [28]

$K=\left|\frac{\partial \log _{10} T_{\alpha(S)}}{\partial Q_{\alpha}}\right|$.

After the straightforward transformations, relation (9) becomes

$$
\begin{aligned}
& K=\left|\frac{\partial \log _{10} T_{\alpha(S)}}{\partial Q_{\alpha}}\right|=\mid-\frac{1}{2}(a)\left(Z_{p}-2\right)\left(Q_{\alpha}\right)^{3 / 2}- \\
& -c A Z N\left[l(l+1)^{1 / 4} Q_{\alpha}^{-2} \mid,\right.
\end{aligned}
$$

for $\mathrm{e}^{-} \mathrm{e}, \mathrm{e}^{-} \mathrm{O}, \mathrm{o}^{-} \mathrm{e}$, and $\mathrm{o}^{-} \mathrm{O}$ nuclei, where $a, l$, and $c$ are the same as above. Formula (10) helps us to explain 
the energy dependence of the alpha-decay half-life. To reveal the behavior of $K$ values more obviously, we determine the $K$ data for all types of superheavy elements ranging from $Z=100$ to $Z=120$. Moreover, we will calculate the difference between the experimental $T_{1 / 2}^{\exp }$ and theoretical $T_{1 / 2}^{\text {theo }}$ values for $\alpha$-decays:

$\Delta T=\left(\log _{10} T_{1 / 2}^{\text {exp }}\right)-\left(\log _{10} T_{1 / 2}^{\text {theo }}\right)$.

In order to measure the deviation of the obtained data, we go in the standard way. The RMS deviations are determined by [31]:

$\sigma=\left\{\frac{1}{N} \sum_{i=1}^{N}\left[\left(\left(\log _{10} T_{1 / 2}^{\mathrm{exp}}\right)-\left(\log _{10} T_{1 / 2}^{\text {theo }}\right)\right)^{2}\right]\right\}^{1 / 2}$,

where $T_{1 / 2}$ theo is the theoretical value of the alphadecay half-life and $N_{\text {tot }}$ is the total number of all nuclei under study that decay with the emission of an alpha particle $\left(N_{\text {tot }}=128\right)$. The determined values of RMS deviations for the three models (Viola-Seaborg, G. Royer, and Present Work Model) for all types of nuclei are shown in Table 5.

\section{Results and Discussion}

The properties of the alpha decay of 128 superheavy nuclei within the interval $100 \leqslant Z \leqslant 120$ have been studied by evaluating the alpha-decay half-lives using the Viola-Seaborg formula (VSF), Royer formula (RF), and Present Work Formula (PWF). The latter involves the effect of a relative neutron excess [see relation (8)] and the angular momentum $(l)$ of the ejected alpha particle. The alpha emission obeys the spin-parity selection rule:

$\left|I_{p}-I_{d} \leq l \leq I_{p}+I_{d}\right|$ and $\pi_{p}=(-1)^{l} \pi_{d}$,

where $I_{p}, I_{d}$, and $\pi_{p}, \pi_{d}$ are the spins and parities of the mother and daughter nucleus, respectively. Tables 1-4 show the evaluated the alpha-decay half-lives for the three models.

A comparative calculation for the standard deviations of $\mathrm{e}^{-} \mathrm{e}, \mathrm{e}-\mathrm{O}, \mathrm{O}^{-} \mathrm{e}$, and $\mathrm{O}^{-} \mathrm{O}$ nuclei in the alpha transitions for (VSF), (RF), and (PWF) are listed in Table 5 that shows the most accurate and best alphadecay half-lives. The first column represents the types of nuclei. The second, third, and fourth columns identify the standard deviations for the (VSF), (RF), and (PWF) models. The last column represents the number of mother nuclei.
Table 1. The predicted $\log _{10} T_{1 / 2}^{\text {theo }}$

for (VSF), (RF), (PWF) and $\log _{10} T_{1 / 2}^{\text {exp }}$

of even-even nuclei in the interval $100 \leq Z \leq 120$

\begin{tabular}{|c|c|c|c|c|c|}
\hline $\begin{array}{l}\text { Nuc- } \\
\text { leus }\end{array}$ & $Z, N, A$ & $\begin{array}{c}\log _{10} T_{1 / 2}^{\text {theo }} \\
\text { (VSF) }\end{array}$ & $\begin{array}{c}\log _{10} T_{1 / 2}^{\text {theo }} \\
(\mathrm{RF})\end{array}$ & $\begin{array}{c}\log _{10} T_{1 / 2}^{\text {theo }} \\
(\mathrm{PWF})\end{array}$ & $\log _{10} T_{1 / 2}^{\exp }$ \\
\hline $\mathrm{Sg}$ & $106,154,260$ & -2.3582 & -2.3132 & -2.1703 & -2.444 \\
\hline $\mathrm{Sg}$ & $106,156,271$ & 1.4438 & 1.2918 & 1.6133 & 2.05 \\
\hline $\mathrm{Sg}$ & $106,160,266$ & 1.1399 & 1.0810 & 1.36 & 1.531 \\
\hline Hs & $108,156,264$ & -2.6485 & -3.2969 & -3.1449 & -3.585 \\
\hline $\mathrm{Hs}$ & $108,162,270$ & -0.0952 & 0.1061 & 0.3952 & 0.556 \\
\hline $\mathrm{Hs}$ & $108,158,266$ & -2.6152 & -2.6144 & -2.4287 & -1.91 \\
\hline $\mathrm{Hs}$ & $108,160,268$ & -1.4483 & -1.4831 & -1.2518 & -0.69 \\
\hline Hs & $108,164,272$ & -1.9881 & -2.0998 & -1.8554 & -1.33 \\
\hline Hs & $108,166,274$ & -0.318 & -0.4634 & -0.1602 & 0.44 \\
\hline Hs & $108,168,276$ & 1.8749 & 1.6971 & 2.0732 & 2.77 \\
\hline 114 & $114,172,286$ & -0.792 & -0.937 & -0.5413 & -0.886 \\
\hline 114 & $114,174,288$ & -0.1195 & -0.3016 & 0.1261 & -0.097 \\
\hline 116 & $116,174,290$ & -1.9248 & -2.0617 & -1.6632 & -2.167 \\
\hline 116 & $116,176,292$ & -1.4569 & -1.6312 & -1.2064 & -1.745 \\
\hline 118 & $118,176,294$ & -3.3151 & -3.4384 & -3.0457 & -3.046 \\
\hline 118 & $118,166,284$ & -5.2459 & -5.1789 & -4.908 & -4.62 \\
\hline 118 & $118,168,286$ & -4.3803 & -4.3528 & -4.0436 & -3.72 \\
\hline 118 & $118,170,288$ & -3.425 & -3.4369 & -3.0869 & -2.72 \\
\hline 118 & $118,172,290$ & -2.8218 & -2.8723 & -2.4916 & -2.11 \\
\hline 120 & $120,178,298$ & -4.4471 & -4.5532 & -4.159 & -4.523 \\
\hline 120 & $120,154,274$ & -11.8684 & -11.498 & -11.4832 & -11.52 \\
\hline 120 & $120,156,276$ & -11.143 & -10.8138 & -10.7636 & -10.28 \\
\hline 120 & $120,160,280$ & -8.9085 & -8.6632 & -8.5199 & -8.4 \\
\hline 120 & $120,162,282$ & -7.6293 & -8.4262 & -7.2318 & -7.05 \\
\hline 120 & $120,164,284$ & -4.5723 & -4.4159 & -4.1193 & -3.78 \\
\hline 120 & $120,166,286$ & -6.1101 & -5.9875 & -5.7215 & -5.5 \\
\hline $\mathrm{Fm}$ & $100,150,250$ & 3.2163 & 3.2941 & 3.4869 & 3.38 \\
\hline $\mathrm{Fm}$ & $100,146,246$ & 0.3338 & 0.4718 & 0.5612 & 0.17 \\
\hline $\mathrm{Fm}$ & $100,148,248$ & 1.5988 & 1.7055 & 1.8422 & 1.66 \\
\hline $\mathrm{Fm}$ & $100,154,254$ & 4.1851 & 4.1919 & 4.4385 & 4.14 \\
\hline $\mathrm{Fm}$ & $100,156,256$ & 4.418 & 4.3881 & 4.6547 & 4.405 \\
\hline No & $102,156,258$ & 1.85 & 1.8222 & 2.0462 & 2.08 \\
\hline No & $102,150,252$ & 0.5152 & 0.596 & 0.7422 & 0.74 \\
\hline No & $102,152,254$ & 1.5889 & 1.6365 & 1.8252 & 1.82 \\
\hline $\mathrm{Rf}$ & $104,152,256$ & -0.0228 & 0.0406 & 0.2095 & -0.52 \\
\hline $\mathrm{Rf}$ & $104,154,258$ & -0.9207 & -0.8997 & -0.7401 & -1.04 \\
\hline $\mathrm{Rf}$ & $104,156,260$ & 0.1281 & 0.1151 & 0.3167 & 0.00 \\
\hline Ds & $110,152,262$ & -5.6376 & -5.4879 & -5.4072 & -5.05 \\
\hline Ds & $110,154,264$ & -5.3594 & -5.2483 & -5.1454 & -4.79 \\
\hline Ds & $110,156,266$ & -4.9444 & -4.8714 & -4.7428 & -4.37 \\
\hline Ds & $110,158,268$ & -5.68 & -5.6466 & -5.5239 & -5.18 \\
\hline Ds & $110,160,270$ & -4.0828 & -4.0855 & -3.905 & -3.49 \\
\hline 112 & $112,152,264$ & -6.7267 & -6.5345 & -6.4641 & -6.18 \\
\hline 112 & $112,154,266$ & -6.5888 & -6.4356 & -6.3468 & -6.07 \\
\hline 112 & $112,156,268$ & -6.0867 & -5.9722 & -5.8551 & -5.56 \\
\hline 112 & $112,158,270$ & -5.5902 & -5.5141 & -5.369 & -5.06 \\
\hline 112 & $112,160,272$ & -5.122 & -5.0841 & -4.9118 & -4.56 \\
\hline 112 & $112,162,274$ & -4.5962 & -4.5962 & -4.3954 & -4.02 \\
\hline 112 & $112,164,276$ & -3.8229 & -3.8605 & -3.6244 & -3.22 \\
\hline 112 & $112,166,278$ & -3.3277 & -3.4047 & -3.1392 & -2.72 \\
\hline
\end{tabular}


Table 2. The predicted $\log _{10} T_{1 / 2}^{\text {theo }}$

for (VSF), (RF), (PWF) and $\log _{10} T_{1 / 2}^{\text {exp }}$

of even-odd nuclei in the interval $100 \leq Z \leq 120$

\begin{tabular}{|c|c|c|c|c|c|}
\hline Nuc- & $Z, N, A$ & $\begin{array}{c}\log _{10} T_{1 / 2}^{\text {theo }} \\
\text { leus }\end{array}$ & $\begin{array}{c}\log _{10} T_{1 / 2}^{\text {theo }} \\
(\mathrm{RF})\end{array}$ & $\begin{array}{c}\log _{10} T_{1 / 2}^{\text {theo }} \\
(\mathrm{PWF})\end{array}$ & $\log _{10} T_{1 / 2}^{\text {exp }}$ \\
\hline Rf & $104,151,255$ & 1.034 & 0.8576 & 0.4576 & 0.204 \\
Rf & $104,155,259$ & 0.6727 & 0.4212 & 0.0994 & 0.519 \\
Rf & $104,157,261$ & 1.8395 & 1.5611 & 1.4128 & 1.86 \\
Sg & $106,155,261$ & -0.6066 & -0.8168 & -1.2555 & -0.638 \\
Sg & $106,157,263$ & 0.2847 & 0.0445 & -0.2522 & -0.523 \\
Sg & $106,159,265$ & 1.5627 & 1.2954 & 1.1841 & 0.851 \\
Sg & $106,165,271$ & 2.6472 & 2.2813 & 2.463 & -3.097 \\
Hs & $108,157,265$ & -2.6125 & -2.8158 & -3.3849 & -1.481 \\
Hs & $108,159,267$ & -0.8315 & -1.0604 & -1.4042 & 0.987 \\
Hs & $108,161,269$ & 1.0269 & 0.7732 & 0.6724 & -5.523 \\
Ds & $110,157,267$ & -4.4074 & -44.5599 & -5.2888 & -3.77 \\
Ds & $110,159,269$ & -3.3119 & -3.495 & -4.0709 & -2.959 \\
Ds & $110,161,271$ & -2.4185 & -2.633 & -3.0708 & -3.77 \\
Ds & $110,163,273$ & -3.5050 & -3.7603 & -4.2522 & -3.77 \\
Ds & $110,169,279$ & 0.3304 & -0.0115 & 0.0536 & -0.699 \\
Cn & $112,171,283$ & 1.4541 & 1.1412 & 1.3961 & 0.58 \\
114 & $114,173,287$ & 0.7178 & 0.4291 & 0.6706 & -0.319 \\
114 & $114,175,289$ & 1.292 & 0.9707 & 1.3363 & 0.415 \\
118 & $118,177,295$ & -2.0134 & -2.2432 & -2.1695 & -3.000 \\
120 & $120,179,299$ & -3.1627 & -3.3555 & -3.3424 & -4.301 \\
No & $102,151,253$ & 2.8091 & 2.6019 & 2.3667 & 1.982 \\
No & $102,153,255$ & 2.4345 & 2.1879 & 1.9751 & 2.27 \\
No & $102,155,257$ & 1.6975 & 1.4085 & 1.1814 & 1.398 \\
No & $102,157,259$ & 4.0127 & 3.7082 & 3.7764 & 3.542 \\
No & $102,149,251$ & 0.9795 & 0.7928 & 0.3249 & 0.00 \\
Fm & $100,145,245$ & 1.1994 & 1.0455 & 0.4796 & 0.62 \\
Fm & $100,147,247$ & 2.0214 & 1.8387 & 1.4056 & 2.07 \\
Fm & $100,149,249$ & 3.3457 & 3.1394 & 2.8911 & 2.59 \\
Fm & $100,151,251$ & 6.7774 & 6.5684 & 6.7255 & 6.07 \\
Fm & $100,153,253$ & 6.3087 & 6.059 & 6.2333 & 6.70 \\
\hline & & & & \\
\hline
\end{tabular}

According to the value of $\sigma$ in Table 5, (PWF) and (RF) can be considered the best models for $\mathrm{o}^{-} \mathrm{e}$ and e-o nuclei compared with (VSF); (VSF) is the best for $\mathrm{O}^{-\mathrm{O}}$ nuclei; while (PWF) shows more acceptable results, than (VSF) and (RF). The results of evaluation of the overall effect of proton and neutron shells on the alpha-decay half-life, which allows us to judge the nucleus stability, are shown in Figs. 1, $a, b, c$, $d, 2 a, b, c, d$, and $3, a, b, c, d$. The $\Delta T$ logarithm versus the neutron number $(N)$ curves were calculated with the use of (VSF), (RF), and (PWF) for the even-even, even-odd, odd-even, and odd-odd nuclei emitting alpha particles. These figures show the acceptable results.
Table 3. The predicted $\log _{10} T_{\mathbf{1} / \mathbf{2}}^{\text {theo }}$

for (VSF), (RF), (PWF) and $\log _{10} T_{1 / 2}^{\text {exp }}$

of odd-even nuclei in the interval $100 \leq Z \leq 120$

\begin{tabular}{|c|c|c|c|c|c|}
\hline $\begin{array}{c}\text { Nuc- } \\
\text { leus }\end{array}$ & $Z, N, A$ & $\begin{array}{c}\log _{10} T_{1 / 2}^{\text {theo }} \\
(\mathrm{VSF})\end{array}$ & $\begin{array}{c}\log _{10} T_{1 / 2}^{\text {theo }} \\
(\mathrm{RF})\end{array}$ & $\begin{array}{c}\log _{10} T_{1 / 2}^{\text {theo }} \\
(\mathrm{PWF})\end{array}$ & $\log _{10} T_{1 / 2}^{\text {exp }}$ \\
\hline $\mathrm{Db}$ & $105,152,257$ & -0.0914 & -0.5102 & -0.4342 & -0.097 \\
$\mathrm{Db}$ & $105,156,260$ & 0.604 & 0.1331 & 0.3186 & 0.255 \\
$\mathrm{Bh}$ & $107,160,267$ & 1.6232 & 1.1001 & 1.4842 & 1.230 \\
$\mathrm{Mt}$ & $109,166,275$ & -1.9844 & -2.6002 & -2.3065 & -2.013 \\
$\mathrm{Mt}$ & $109,158,267$ & -2.94 & -3.41 & -3.34 & -3.33 \\
$\mathrm{Mt}$ & $109,160,269$ & -2.1103 & -2.61 & -2.3 & -1.47 \\
$\mathrm{Mt}$ & $109,162,271$ & -0.48 & -1.0168 & -0.07 & -0.16 \\
113 & $113,172,258$ & 0.9029 & 0.7905 & 0.8527 & 0.739 \\
113 & $113,174,287$ & 1.8 & 1.2 & 1.9 & 1.6 \\
115 & $115,172,287$ & -0.8100 & -1.3915 & -0.8268 & -1.456 \\
115 & $115,174,289$ & 0.0610 & -0.6768 & -0.0138 & -0.658 \\
115 & $115,176,291$ & 0.29 & -0.3 & 0.38 & -0.18 \\
115 & $115,182,297$ & 3.14 & 2.3 & 3.4 & 3.48 \\
117 & $117,176,293$ & -1.3326 & -1.936 & -1.3005 & -1.824 \\
$\mathrm{Lr}$ & $103,152,255$ & 1.4687 & 0297 & 1.1838 & 1.338 \\
$\mathrm{Lr}$ & $103,154,257$ & 0.1158 & -0.3723 & -0.2590 & -0.187 \\
$\mathrm{Lr}$ & $103,156,259$ & 1.4430 & 0.9280 & 1.1706 & 0.792 \\
111 & $111,160,271$ & -3.86 & -4.3321 & -4 & -3.08 \\
111 & $111,168,279$ & -1.3441 & -1.94 & -1.74 & -2.67 \\
111 & $111,164,275$ & -4.340 & -4.89 & -4 & -3.49 \\
111 & $111,162,273$ & -4.5 & -4.1 & -3.4 & -3.87 \\
\hline
\end{tabular}

We can note a decrease in the alpha-decay half-lives with an increase in $Z$, on the whole. But they increase with the neutron number for isotopes of a specific element. The local maximum appears for even- $Z$ nuclei with $N=156$ and $N=151$. It is connected with an increasing of the stability in the vicibity of the so-called distorted magic number of neutrons, this result being in agreement with [8]. The alpha-decay half-lives increase with $N$ beyond 146 and up to 158 for all elements. In these nuclei (especially for superheavy ones), the alpha decay would be irrelevant decay channel under the spontaneous fission. it may have a low half-live. In deed, the curves plotted for lower $Z \mathrm{~s}$ in Fig. $4, a, b, c, d$ show that the peak at $N=156$ and $Z=100$ is higher than the peak appeared for $N=172$ and $Z=112$, while the discontinuities can be interpreted as the effect which appears due to the closed shell structure. The distance between the peaks for the atomic numbers equal to 108 and 110 are of special interest. The same behavior can be observed for the half-lifes of other isotopic chains described by (VSF) and (RF) for all types of 

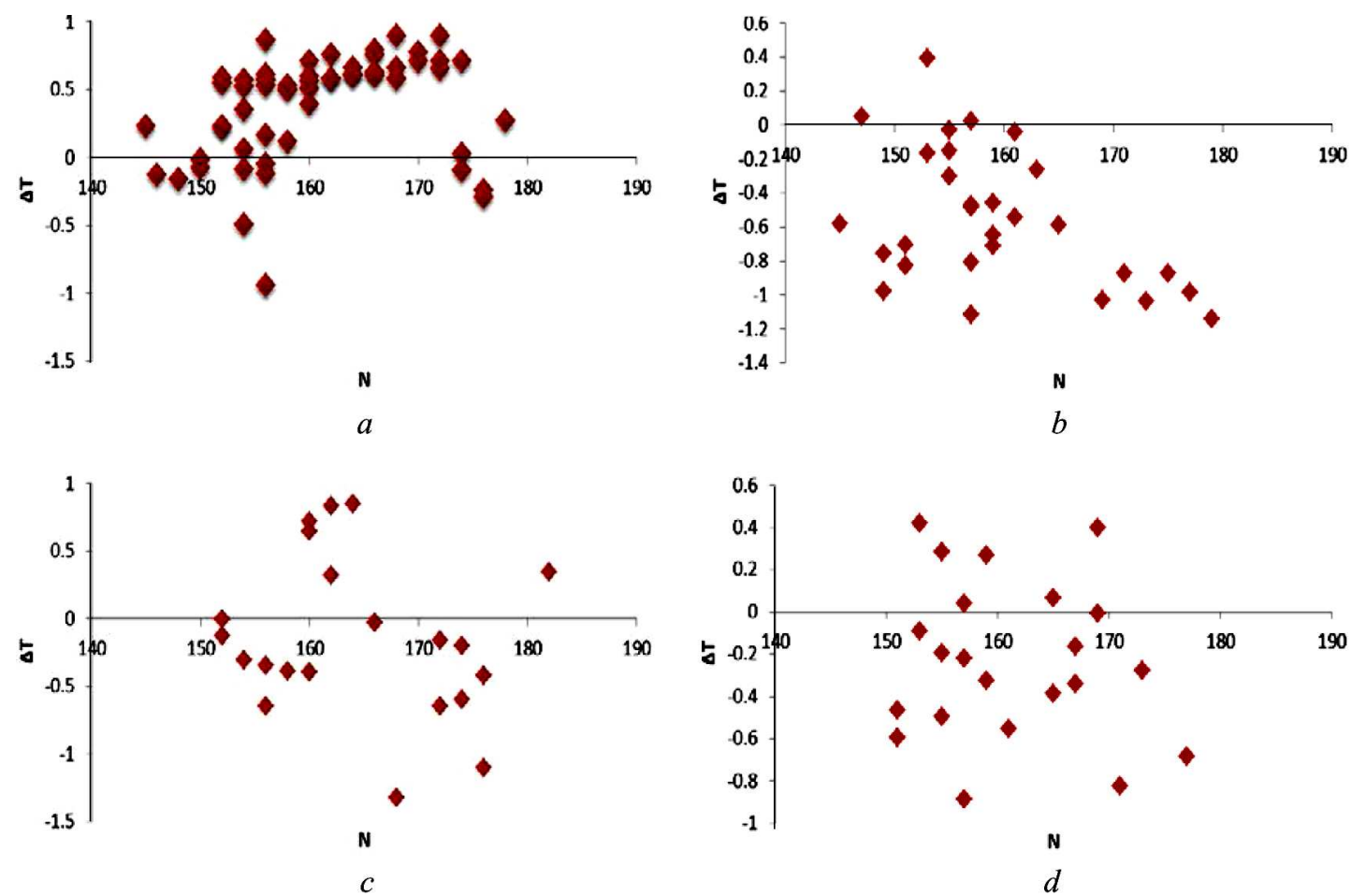

Fig. 1. The disparities between experimental and calculated alpha-decay half-lives for e--e, e--o, o-e, and o--o nuclei versus $N$ for (VSF)
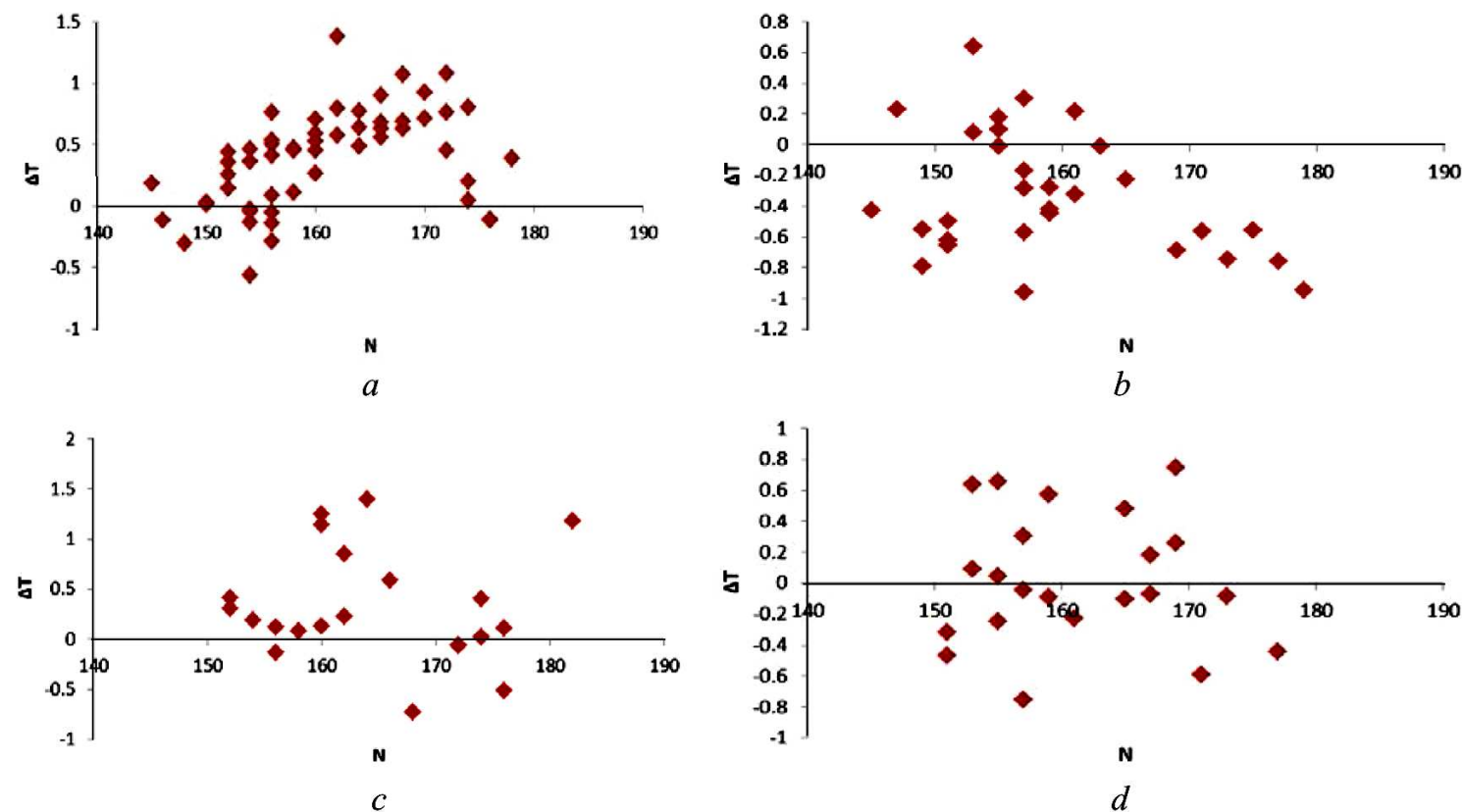

Fig. 2. The disparities between experimental and calculated alpha-decay half-lives for $\mathrm{e}^{-}-\mathrm{e}, \mathrm{e}-\mathrm{o}, \mathrm{o}-\mathrm{e}, \mathrm{and} \mathrm{o}-\mathrm{o}$ nuclei versus $N$ for (RF) 

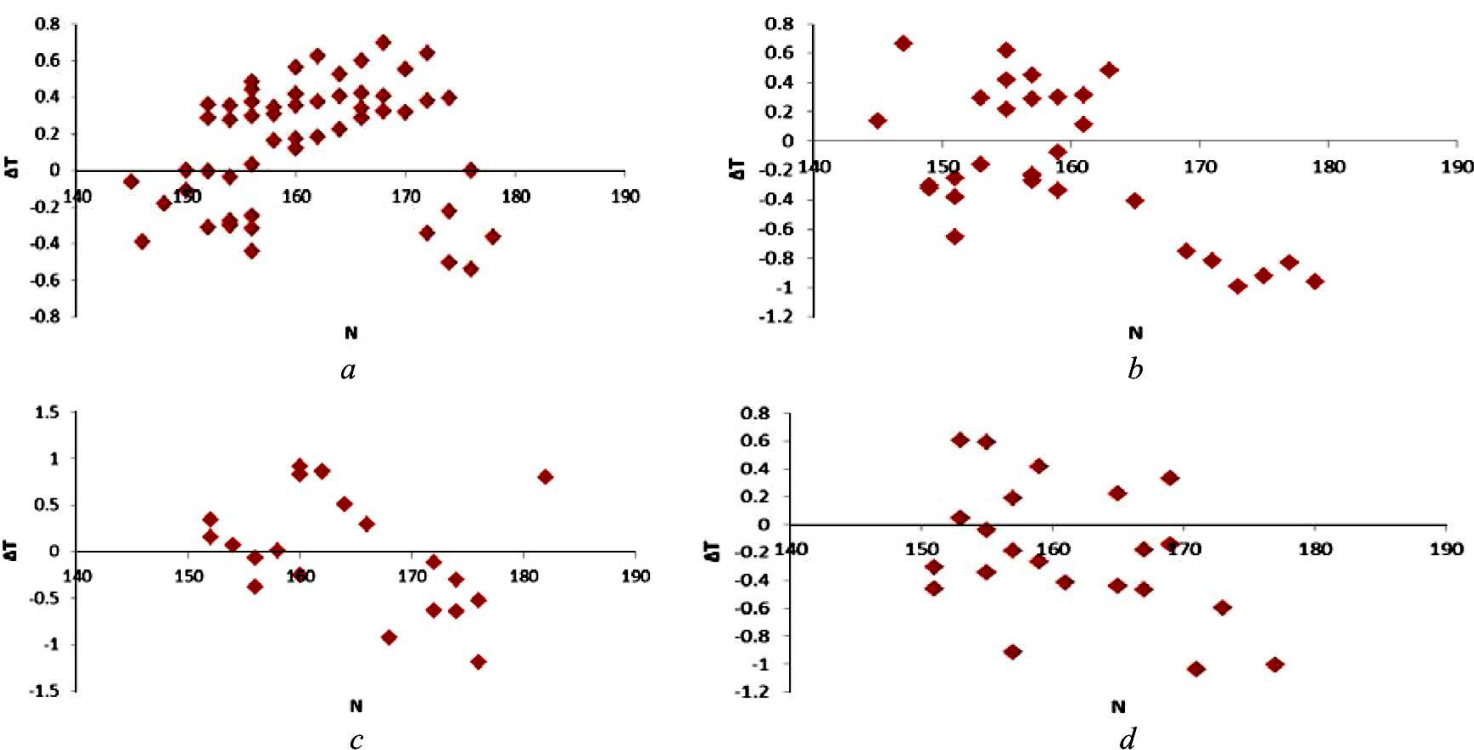

Fig. 3. The disparities between practical and calculated half-lives of alpha decay for e-e, e-o, o-e and o-o nuclei versus $N$ for $(\mathrm{PWF})$
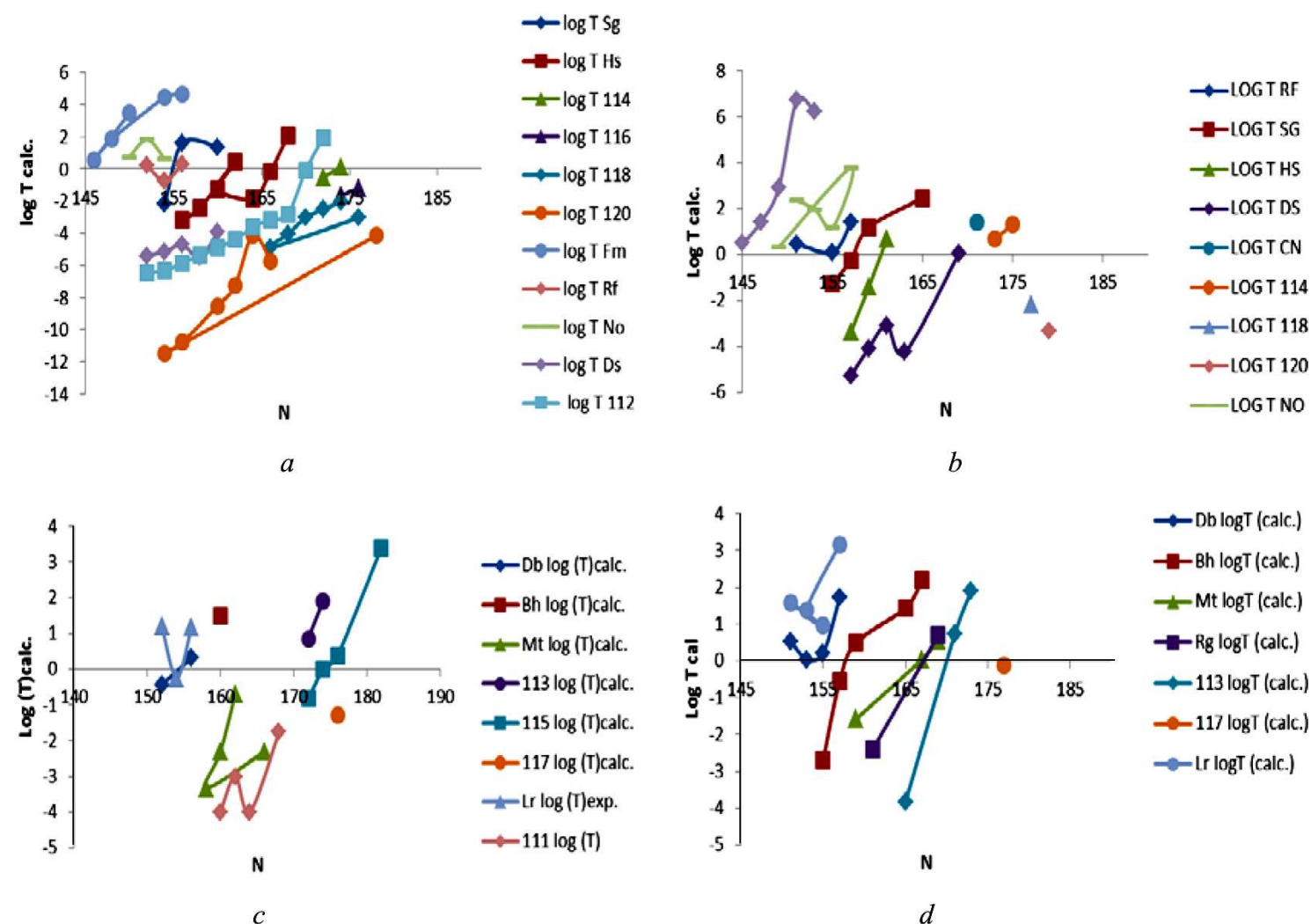

$\rightarrow \mathrm{Db} \log \mathrm{T}$ (calc.)

$\rightarrow-B h \log T$ (calc.)

\pm Mt $\log T$ (calc.)

$\rightarrow-R g \log T$ (calc.)

$\multimap 113 \log T$ (calc.)

$\leadsto 117 \log T$ (calc.)

-- Ir logT (calc.)

$c$

Fig. 4. The theoretical alpha-decay half-lives of $\mathrm{e}-\mathrm{e}, \mathrm{e}-\mathrm{O}, \mathrm{o}-\mathrm{e}$, and $\mathrm{o}-\mathrm{O}$ nuclei, respectively, as a function of the neutron number $N$ for (PWF) only 

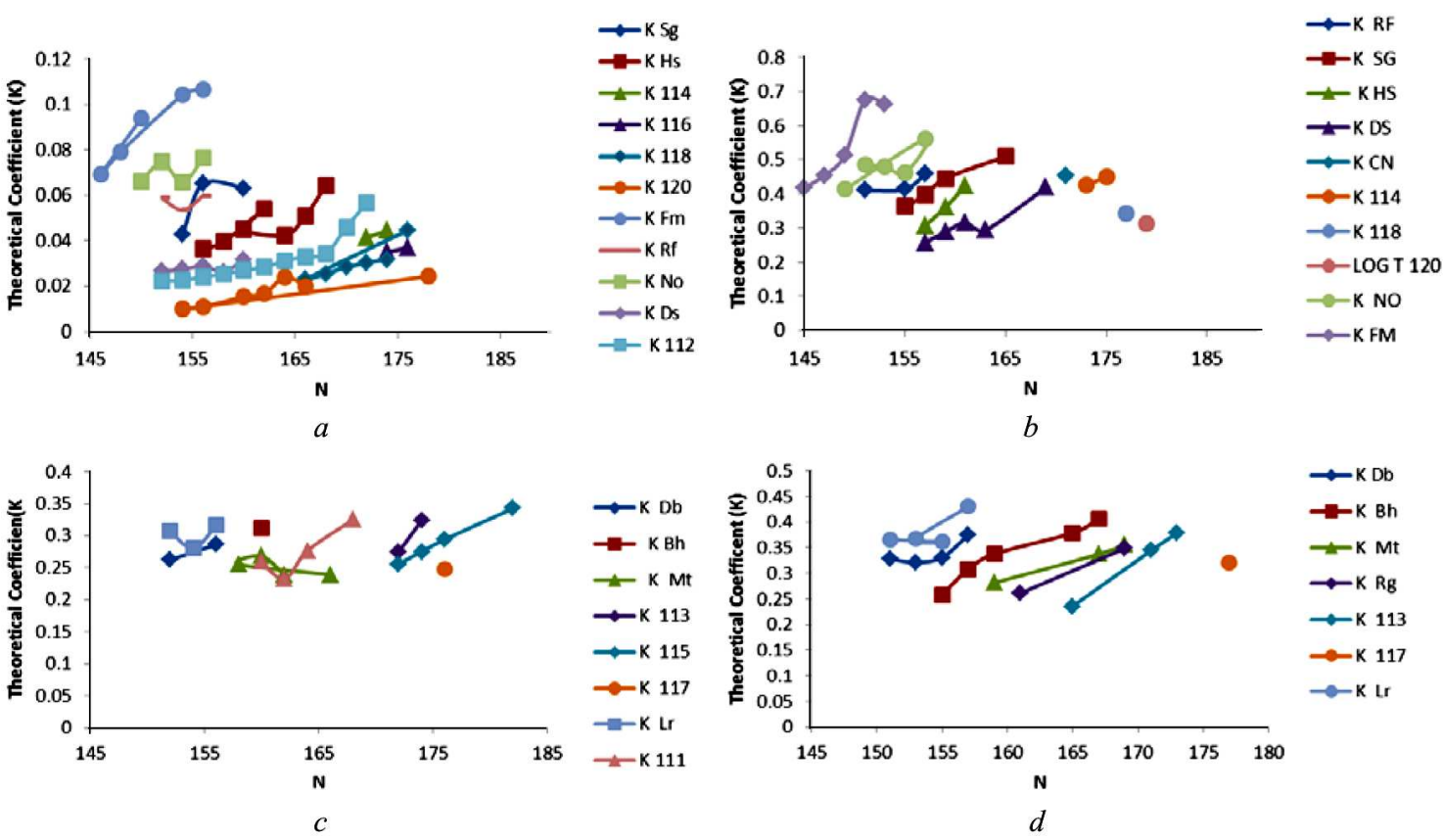

Fig. 5. $K$ for $\mathrm{e}^{-} \mathrm{e}, \mathrm{e}-\mathrm{O}, \mathrm{O}^{-} \mathrm{e}$, and $\mathrm{o}-\mathrm{O}$ nuclei versus the neutron number $N$

Table 4. The predicted $\log _{10} T_{1 / 2}^{\text {theo }}$

for (VSF), (RF), (PWF) and $\log _{10} T_{1 / 2}^{\text {exp }}$

of odd-odd nuclei in the interval $100 \leq Z \leq 120$

\begin{tabular}{|c|c|c|c|c|c|}
\hline $\begin{array}{c}\text { Nuc- } \\
\text { leus }\end{array}$ & $Z, N, A$ & $\begin{array}{c}\log _{10} T_{1 / 2}^{\text {theo }} \\
(\mathrm{VSF})\end{array}$ & $\begin{array}{c}\log _{10} T_{1 / 2}^{\text {theo }} \\
(\mathrm{RF})\end{array}$ & $\begin{array}{c}\log _{10} T_{1 / 2}^{\text {theo }} \\
(\mathrm{PWF})\end{array}$ & $\log _{10} T_{1 / 2}^{\exp }$ \\
\hline Db & $105,151,125$ & 0.6944 & 0.5511 & 0.5333 & 0.230 \\
$\mathrm{Db}$ & $105,153,258$ & 0.2216 & 0.0062 & 0.0382 & 0.643 \\
$\mathrm{Db}$ & $105,155,260$ & 0.3729 & 0.1317 & 0.2145 & 0.176 \\
$\mathrm{Db}$ & $105,157,262$ & 1.7633 & 1.5876 & 1.7285 & 1.544 \\
$\mathrm{Bh}$ & $107,155,262$ & -2.3807 & -2.7542 & -2.6895 & -2.097 \\
$\mathrm{Bh}$ & $107,159,266$ & 0.5549 & 0.3211 & 0.4936 & -0.356 \\
$\mathrm{Bh}$ & $107,157,264$ & -0.3948 & -0.6609 & -0.5435 & 0.230 \\
$\mathrm{Bh}$ & $107,165,272$ & 1.3796 & 1.0971 & 0.4326 & 0.991 \\
$\mathrm{Bh}$ & $107,167,274$ & 2.0719 & 1.8039 & 2.2009 & 1.733 \\
$\mathrm{Mt}$ & $109,159,268$ & -1.4212 & -1.7258 & -1.5758 & -1.5758 \\
$\mathrm{Mt}$ & $109,167,276$ & 0.0217 & -0.3243 & 0.0361 & 0.0361 \\
$\mathrm{Mt}$ & $109,169,278$ & 0.4819 & 0.1332 & 0.5505 & 0.5505 \\
$\mathrm{Rg}$ & $111,161,272$ & -2.2686 & -2.5936 & -2.4113 & -2.4113 \\
$\mathrm{Rg}$ & $111,169,280$ & 0.5637 & 0.2944 & 0.7003 & 0.7003 \\
113 & $113,165,278$ & -3.6831 & -4.0999 & -3.8418 & -3.8418 \\
113 & $113,171,284$ & 0.5031 & 0.2708 & 0.7159 & 0.7159 \\
113 & $113,173,286$ & 1.5686 & 1.3752 & 1.8892 & 1.8892 \\
117 & $117,177,294$ & -0.4317 & -0.6712 & -0.1703 & -0.1073 \\
$\mathrm{Lr}$ & $103.151,254$ & 1.7112 & 1.5797 & 1.5769 & 1.5769 \\
$\mathrm{Lr}$ & $103,155,258$ & 1.1093 & 0.8588 & 0.9577 & 0.9577 \\
$\mathrm{Lr}$ & $103,153,256$ & 1.52 & 1.3371 & 1.3857 & 1.3857 \\
$\mathrm{Lr}$ & $103,157,260$ & 3.1422 & 3.007 & 3.1705 & 3.1705 \\
\hline
\end{tabular}

nuclei. Tables $1-4$ show the acceptable agreement between the analytical determinations and the experimental values, which is a good indicator for the guessing for the alpha-decay half-lives of 121 nuclei within all models that are used. The PWF can be verified in a wide region of nuclear structures, as well as can be applied to the comparison between applied and theoretical nuclear models. To show the meaning of the theoretical coefficient $K$ more clearly, we determine it for superheavy elements ranging from $Z=10$ to $Z=120$, as shown in Fig. 5 .

It has been found that the $K$ value decreases, as $Z$ increases, and increases with $N$ for isotopes of a particular element. This means that it has the same behavior as $\log _{10} T_{1 / 2}^{\text {theo }}$ and hints that the half-lives becomes more and more insensitive to the alpha-decay

Table 5. The standard deviation

for (VSF), (RF), and (PWF) within the new approach with new parameters

\begin{tabular}{|c|c|c|c|c|}
\hline Set & $\sigma(\mathrm{VSF})$ & $\sigma(\mathrm{RF})$ & $(\mathrm{PWF})$ & No of nuclei \\
\hline even-even & 0.541 & 0.55 & 0.38 & 55 \\
even-odd & 0.68 & 0.51 & 0.51 & 30 \\
odd-even & 0.53 & 0.64 & 0.52 & 21 \\
odd-odd & 0.43 & 0.43 & 0.58 & 22 \\
\hline
\end{tabular}


energy. For instance, the decrease in the $Q_{\alpha}$ value by $2 \mathrm{MeV}$ corresponds to an increase of the half-life by $\approx 8$ orders for isotopes of $110 D_{S}$ (Table 1 ). It is a good advantage to predict the alpha-decay half-lives for superheavy nuclei, because they are not so sensitive to energy decay value as for medium-heavy nuclei [28]. For nuclei near the closed shell, the $K$ values are low, because they are affected by the closed shell structure, especially for a heavy element with low $\log _{10} T_{1 / 2}$. This fact proposes that, for a given massive element, the isotopes at the beginning of the closed shell are more insensitive to $Q_{\alpha}$-value.

\section{Conclusion}

We have investigated the alpha-decay half-lives of e$\mathrm{e}, \mathrm{e}^{-} \mathrm{O}, \mathrm{O}^{-} \mathrm{e}$, and $\mathrm{o}^{-} \mathrm{O}$ superheavy nuclei with use of the Viola-Seaborg-Sobiczewoski formula, Royer formula, and present-work formula with the account for the angular momentum of an alpha particle and the relative neutron excess $\left(\frac{N-Z}{A}\right)$, which is extremely important for the evaluation of the half-live logarithm. The formula we have proposed has a new set of parameters determined by the least square fitting method for the alpha decay of 128 nuclei. We have obtained the standard deviations for each formula and the disparity between the experimental and calculated alphadecay half-lives for all types of nuclei. The obtained results are compared with the corresponding experimental values, and it is revealed that they show a good matching. The proposed theoretical coefficient $K$ shows a similar behavior of $\log _{10} T_{1 / 2}$, which can be used to predict new superheavy nuclei. Moreover, the presented formula can be applied to a wide field of physical verifications. But the half-lives of emitters of an alpha particle are insufficient to obtain more information about the nuclear properties and other aspects of superheavy nuclei (such as vibration bands, nuclear isospin, and various nuclear structures).

1. H. Geiger, J. Nuttall. The range of the alpha-particles from various radioactive substance and a relation between range and period of transformation. Philos. Mag. 22, 613 (1911).

2. G. Audi, O. Bresillon, J. Blachot, A.H. Wapstra. The Nubase evaluation of nuclear and decay properties. Nucl. Phys. A 728, 3 (2003).

3. K.P. Santhosh, R.K. Biju, S. Sahadevan. Semi-empirical formula for spontaneous fission half life time. Nucl. Phys. A 832, 220 (2010).

4. K. Van de Vel. Fine structure in the $\alpha$ decay of ${ }^{188,192}$ Po. Phys. Rev. C 68, 054311 (2003).
5. A. Zdeb, M. Warda, K. Pomorski. Half-lives for $\alpha$ and cluster radioactivity within a Gamow-like model. Phys. Rev. C 87, 024308 (2013).

6. A. Zdeb, M. Ward, K. Pormorski. Half-lives for $\alpha$ and cluster radioactivity in a simple model. Phys. Scr. T 154, 014029 (2013).

7. A. Parkhomenko, A. Sobieczwski. Phenomenological formula for alpha-decay half-lives of heaviest nuclei. Acta. Phys. Pol. B 36, 3095 (2005).

8. A. Pobrowolski, B. Nerlo-Pomorska, K. Pomorski, J. Bartel. On the possibility to observe new shape isomeric in the Po-Th region. Acta. Phys. Pol. B 40, 705 (2009).

9. F.M. Ali, M.I. Khalil. To establish a semi - experimental relationship in order to the determine the half-lives of eveneven heavy nuclei that emit alpha-particles for the range of $82 \leqslant Z \leqslant 102$. Iraqi J. of Sci. 1, 750 (2015) (In Arabic).

10. S. Hofmann, G. Munzenberg. Discovery of the heaviest element. Rev. Mod. Phys. 72, 733 (2000).

11. G. Royer, R.A. Gherghescu. On the formation and alpha decay of super heavy elements. Aunc. Phys. A 699, 479 (2002).

12. D. Zhang, Ch.Fu. Mu. Description of mixed symmetry status in ${ }^{96} \mathrm{Ru}$ using IBM-2. Chin. Phys. Mech. Astro. 60, Article number: 042011 (2017).

13. V.E. Viola Dr., G.T. Seaborg. On the production of nucleides with $A>250$ in stellar nuclosynthesis. J. Inorg. Nucl. Chem. 28, 741 (1966).

14. D.N. Poenaru, M. Ivascu. Estimation of the alpha decay half-lives. J. Phys. G. Nucl. Part. Phys. 44, 791 (1983).

15. D.T. Akrawy, H. Hassanabadi, S.S. Hhosseini, K.P. Santhosh. Nuclear isospin effect on $\alpha$-decay half-lives. $\mathrm{Nucl}$. Phys. A 975, 19 (2018).

16. A. Armbruster,Annu. On the production of superheavy nuclei in reactions of ${ }_{20}^{48} \mathrm{Ca}$ with the Heavies actinide targets. Rev. Nucl. Part. Sci. 50, 411 (2000).

17. S. Hofmann et al. New results on elements 111 and 112 . Eur. Phys. J. A 14, 147 (2002).

18. M. Sayed, S. ALmadar. Estimation of alpha decay half-lives for isotopes superheavy nuclei (even $[Z]-$ even $[N]$, (evenodd), (odd-even) and (odd-odd) the range atomic number $Z=104-118$. Faculty of science. Cumhuriyet university. Sci. J. (CSJ) 36 (3), special Issue (2015).

19. Y.Z. Wang, S.J. Wang, Z.Y. Hou, J.Z. Gu. Systematic study of alpha decay energies and half-lives of super heavy nuclei. Phys. Rev. C 92, 064301 (2015).

20. D.N. Poenaru, R.A. Gherghescu, N. Carjan. Alpha-decay life times semi empirical relationship including shell effects. Euro Phys. Lett. 77, 62001 (2007).

21. A. Sobicczewski, A. Parkhomenko. Description of structure and properties of super heavy nuclei. Prog. Part. Nucl. Phys. 58, 292 (2007).

22. D.Ni, Z. Ren, T. Dong, C. Xu. Unified formula of half-lies for alpha decay and cluster radioactivity. Phys. Rev. C 78, 044310 (2008).

23. I. Silisteanu, C.I. Anghel. Alpha-decay and spontaneous fission half lives of super heavybnuclei around the double magic nucleus ${ }^{270} \mathrm{Hs}$. EJP Web of Conference 107, 07004 (2016).

ISSN 2071-0194. Ukr. J. Phys. 2020. Vol. 65, No. 2 
24. G. Royer. Alpha emission and spontaneous fission through quasi-molecular shape. J. Phys. G. Nucl. Part. Phys. 26, 1149 (2000).

25. S.S. Malik, R.K. Gupta. Theory of cluster radioactive decay and of cluster formation in nuclei. Phys. Rev. C 39, 1992 (1989).

26. H. Koura. Alpha-decay half-lives and fission barrier for superheavy nuclei predicted by a nuclear mass formula. J. Nucl. And Radiochemical. Sci. 3, (1) (2002).

27. V.Yu. Denesov, A.A. Khudenko. Alpha-decay half-lives: Empirical relations. Phys. Rev. C 79, 054614 (2009).

28. J. Dong, W. Zuo, J. Gu, Y. Wang, B. Peng. $\alpha$-decay halflives and $Q_{\alpha}$ values of superheavy nuclei. Phys. Rev. C 81, 064309 (2010).

29. N.K. Dhiman. Role different model ingredients in the exotic cluster-decay of ${ }_{56} \mathrm{Ni}$. Ukr. J. Phys. 57 (8), (2010).

30. M.O. Waheed, F.I. Sherrad. Description of the deformation properties of even-even ${ }^{102-106} \mathrm{Pd}$ isotopes. Uk. J. Phys. 62 (9), (2017).

31. D. Ni, Z. Ren. Microscopic calculation of alpha-decay halflives within the cluster model. Nucl. Phys. A 825, 145 (2009)

Received 04.07.19
Ф.М.А. Ал-Джсмейлі, М.І. Халіл

АНАЛІЗ СПІВВІДНОШЕННЯ

ГЕЙГЕРА-НУТТАЛА ДЛЯ ЗАСТОСУВАННЯ

ОЦІНКИ ПЕРІОДІВ НАПІВРОЗПАДУ

НАДВАЖКИХ ЯДЕР

$\mathrm{P}$ е $з$ ю м е

Періоди напіврозпаду парно-парних, парно-непарних, непарно-парних і непарно-непарних ядер в інтервалі $100 \leqslant$ $\leqslant Z \leqslant 120$ оцінювалися за формулою Віола-Сіборга (VSF) і за аналітичною формулою Роєра (RF). Ми запропонували іншу формулу (PWF), яка враховує кутовий момент частинки в альфа-розпаді і відносний нейтронний надлишок $\left(\frac{N-Z}{A}\right)$. Наша формула містить новий набір параметрів, які визначаються за методом найменших квадратів для альфарозпадів 128 ядер. Ми отримали і порівняли стандартні відхилення для кожної з формул. Результати показують прийнятне узгодження 3 наявними даними. Величини запропонованого теоретичного коефіцієнта $K$ в нашій моделі (PWF) показують схожу поведінку періодів напіврозпаду ядер з альфа-розпадом, що може бути використано для передбачення нових надважких ядер. 